\title{
Transfer of Motor Learning across Arm Configurations
}

\author{
Nicole Malfait, ${ }^{1}$ Douglas M. Shiller, ${ }^{1}$ and David J. Ostry ${ }^{1,2}$ \\ 1McGill University, Montreal, Quebec, Canada H3A 1B1, and 2Haskins Laboratories, New Haven, Connecticut 06511
}

It has been suggested that the learning of new dynamics occurs in intrinsic coordinates. However, it has also been suggested that elements that encode hand velocity, and hence act in an extrinsic frame of reference, play a role in the acquisition of dynamics. To reconcile claims regarding the coordinate system involved in the representation of dynamics, we have used a procedure involving the transfer of force-field learning between two workspace locations. Subjects made point-to-point movements while holding a two-link manipulandum. Subjects were first trained to make movements in a single direction at the left of the workspace. They were then tested for transfer of learning at the right of the workspace. Two groups of subjects were defined. For the subjects in group j, movements at the left

With practice, healthy subjects adapt to externally applied forces (Lackner and Dizio, 1994; Shadmehr and Mussa-Ivaldi, 1994) as well as to novel intersegmental dynamics (Sainburg et al., 1999). Moreover, after adaptation, aftereffects can be observed when forces are unexpectedly removed; this has been interpreted as evidence that subjects use anticipatory mechanisms that rely on neural representations of musculoskeletal dynamics. The coordinate system that is used to develop and store this knowledge is central to understanding the nature of this representation.

Patterns of generalization have been examined as a means to dissociate extrinsic from intrinsic encoding. Shadmehr and Mussa-Ivaldi (1994) and Shadmehr and Moussavi (2000) have provided support for a joint- or muscle-based system of coordinates by showing that adaptation to a hand velocity-dependent force field transfers across workspace locations if the experienced torque field is invariant, rather than the forces applied at the hand. However, other studies have reported evidence that is consistent with movement coding in an external frame of reference. In a catching task, Morton et al. (2001) reported complete transfer of training across two arm configurations that are similar in terms of hand displacement during catching but that entail radically different joint torques. Moreover, a pattern of interlimb generalization that is consistent with extrinsic coding has been observed recently in force-field learning by Hemminger et al. (2001).

Questions may be raised regarding the evidence in favor of joint-based learning. Shadmehr and Mussa-Ivaldi (1994) found

\footnotetext{
Received July 31, 2002; revised Aug. 29, 2002; accepted Aug. 29, 2002.

This work was supported by National Institutes of Health Grant DC-04669 from the National Institute on Deafness and Other Communication Disorders, Natural Sciences and Engineering Research Council of Canada, and Le Fonds pour La Formation de Chercheurs et l'Aide à la Recherche.

Correspondence should be addressed to D. J. Ostry, Department of Psychology, McGill University, 1205 Dr. Penfield Avenue, Montreal, Quebec, Canada H3A 1B1. E-mail: ostry@motion.psych.mcgill.ca.

Copyright (C) 2002 Society for Neuroscience 0270-6474/02/229656-05\$15.00/0
}

and right workspace locations were matched in terms of joint displacements. For the subjects in group $\mathrm{h}$, movements in the two locations had the same hand displacements. Workspace locations were chosen such that for group j, the paths (for training and testing) that were identical in joint space were orthogonal in hand space. The subjects in group $\mathrm{j}$ showed good transfer between workspace locations, whereas the subjects in group $h$ showed poor transfer. These results are in agreement with the idea that new dynamics are encoded in intrinsic coordinates and that this learning has a limited range of generalization across joint velocities.

Key words: arm movement; motor learning; dynamics; force field; coordinate system; generalization

that forces at the hand varied in a complex manner, assisting movement in some directions and opposing movement in others. Under such conditions, subjects may have been unable to use the pattern of forces at the hand on which to base learning.

Ghez et al. (2000) also report the transfer of learning in intrinsic coordinates. Subjects adapted to altered intersegmental dynamics, reaching to a single target in one region of the workspace. On selected trials, they were required to move, in another part of the workspace, to either of two targets. One target required the same joint displacements as the training target, whereas the other involved the same hand displacements. Better transfer was found between movements matched for joint excursion.

In the results from Ghez et al. (2000), ambiguity remains as to whether the observed transfer occurred in intrinsic coordinates or in an extrinsic frame of reference. The two transfer directions differed by $22^{\circ}$; with the same loads, substantial transfer across hand movement directions has been reported for movements made $36^{\circ}$ to either side of the trained direction (Sainburg et al., 1999). This means that the direction of the transfer target matched for joint excursion fell within the span of directional generalization of the learning at the level of the hand. As a consequence, transfer attributable to similarity in hand displacement cannot be ruled out.

In the present study, we re-examined the transfer of force-field adaptation using a variant of the approach of Ghez et al. (2000), with the aim of providing a straightforward test that dissociates the learning of dynamics in intrinsic versus extrinsic coordinate frames. The task was designed with several aims in mind. First, it completely dissociates the effects of similarity in joint displacements from similarity in hand direction; training and transfer targets matched for joint displacements were orthogonal in hand space. Second, in contrast to the fields tested by Shadmehr and Mussa-Ivaldi (1994), we used a field that offers a simple pattern of 
forces acting at the hand; for all movement directions the forces are perpendicular to the direction of the movement. As a result, there is nothing that acts to bias the subject against adopting a hand-based learning strategy.

\section{MATERIALS AND METHODS}

Experimental setup. Twenty-four right-handed adults participated in the study. Subjects were seated and held the handle of a two-link manipulandum (Interactive Motion, Cambridge, MA). They made horizontal arm movements with the right arm supported by an air sled. The shoulder was restrained and the wrist was braced. Subjects were instructed to move the handle of the manipulandum to targets that were mounted on a horizontal panel below the apparatus. Full visual feedback was provided.

Experimental procedures. Subjects made $12 \mathrm{~cm}$ point-to-point movements toward $8 \mathrm{~mm}$ diameter targets. The subjects were trained to produce movements of $500 \pm 50 \mathrm{msec}$. A visual display provided feedback on the duration of movement. Movements were performed using two arm configurations, at the left and the right of the workspace. The initial shoulder and elbow angles were $\mathrm{qs}=90^{\circ}$ and $\mathrm{qe}=90^{\circ}$ at the left and $\mathrm{qs}=0^{\circ}$ and $\mathrm{qe}=90^{\circ}$ at the right (the shoulder angle was measured relative to the frontal plane and the elbow angle was measured relative to the upper arm). To avoid differences in inertia associated with changes to the operating configuration of the robot, subjects were moved with respect to the robot for movements in the left and right of the workspace. Two types of dynamic environments were used: (1) a "null field," in which there were no forces applied to the limb, and (2) a force field, in which the force $f$ depended on the velocity of the hand $v: f=B v$, where $B$ is a constant matrix representing viscosity of the imposed environment in hand-end-point coordinates. Specifically, we chose a counterclockwise curl field defined by $B=\{0,-20,20,0\} \mathrm{N} \cdot \mathrm{sec} \cdot \mathrm{m}^{-1}$.

Experiments 1 and 2. Each experiment consisted of a training session at the left of the workspace and a transfer test at the right. In both training and transfer trials, subjects made movements in a single direction such that the directions in the training and transfer trials were similar in either hand or joint coordinates. Thus, some subjects trained in a direction that was similar in hand space to the direction tested in the transfer trials. Other subjects trained in a direction that was similar in joint space to the direction in the transfer trials. The aim was to determine whether the transfer of learning across arm configurations is based on equivalence of movement directions in hand versus joint space.

Figure 1 shows movement directions and associated forces or torques. It also illustrates the fact that curl fields act perpendicular to hand as well as joint trajectories and are invariant under shoulder rotation. The training targets are shown in Figure 1, $A$ and $C$; the transfer targets are shown in Figure $1, B$ and $D$. Figure $1, A$ and $B$, shows the paths of the hand in Cartesian coordinates; Figure $1, C$ and $D$, shows the paths of the joints in angular coordinates. The numbers 1 and 2 represent the directions that were tested in experiments 1 and 2, respectively.

In Figure $1, A$ and $C, \mathrm{j}$ is used to denote training targets that involve the same joint displacements as transfer targets; h labels targets corresponding to the same hand-end-point paths. Thus, for example, movements in direction $1 \mathrm{j}$ in the left workspace are similar in direction in joint space to movements in direction 1 at the right. Movements in direction $1 \mathrm{~h}$ in the left workspace are similar in direction in hand space to movements in direction 1 in the right.

Two separate sets of targets were used in these experiments, set $1=$ $\{1 \mathrm{j}, 1 \mathrm{~h}, 1\}$ and set $2=\{2 \mathrm{j}, 2 \mathrm{~h}, 2\}$, as shown in Figure 1 . In each case, the hand trajectories corresponding to the two training directions were orthogonal to one another. In choosing target directions for these experiments, it would have been desirable to use movements that were orthogonal in direction and of a similar path length in both hand and joint coordinates. This would have minimized any similarity in learning that might have occurred for movements in different directions (attributable to an insufficient angular distance between training directions); at the same time it would have enabled us to equate the forces and torques experienced during training in different directions. However, Figure 1 shows that a complete balancing of the design in this manner is not possible. Accordingly, we have used the two different sets of targets described above, one in which joint paths were orthogonal (set 2) and the other in which joint paths were of similar length (set 1).

Twelve subjects participated in each experiment. They were subdivided into two groups: group $\mathrm{h}$ and group $\mathrm{j}$. In experiment 1 , the subjects in group $\mathrm{h}$ were trained to make movements at the left to target $1 \mathrm{~h}$, which

\section{LEFT}

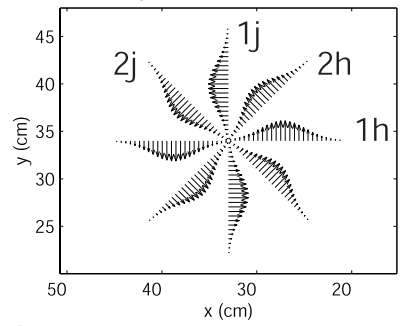

C joint paths

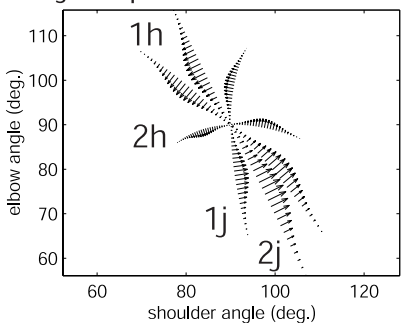

A hand paths
RIGHT

$B$ hand paths

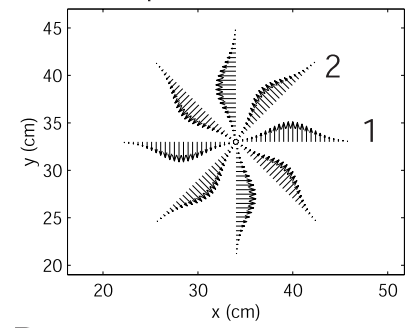

$D$ joint paths

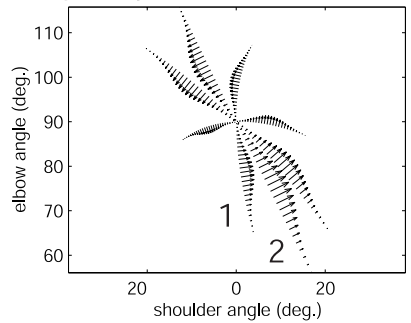

Figure 1. Motion paths and associated forces and torques for movements at the left and right of the workspace. $A$ and $C$ show movement directions used in training trials. $B$ and $D$ show movement directions in transfer trials. 1 and 2 refer to experiments 1 and 2. In $A$ and $C, j$ and $h$ denote training directions that have the same joint or hand paths as the directions tested in the transfer trials shown in $B$ and $D$.

involved the same hand-end-point displacement as transfer target 1 . The subjects in group $\mathrm{j}$ were trained with target $1 \mathrm{j}$, which corresponded to the same joint displacement as target 1 . For experiment 2, the subjects in group $\mathrm{h}$ were trained with target $2 \mathrm{~h}$; the subjects in group $\mathrm{j}$ were trained with target $2 \mathrm{j}$.

Before training, all of the subjects were familiarized with the task by making movements in both workspace locations in the null field, performing first three sets of 20 movements to their training target at the left and then the same number of movements to their transfer target at the right. Subjects were then moved back to their initial position, at the left, where they trained for 20 sets of 20 movements to their training target, while the manipulandum produced the force field. Subjects were given 10 min of rest after the first 10 training sets. At the end of the training, subjects were moved to the right and performed a transfer set of 20 movements under the same force-field conditions.

Data analysis. Hand positions were sampled at $200 \mathrm{~Hz}$, low-pass Butterworth-filtered at $20 \mathrm{~Hz}$, and numerically differentiated. The start and end of movement were defined by $5 \%$ of the maximum tangential velocity. We used three kinematic measures to characterize the adaptation and transfer of learning: (1) the maximum perpendicular displacement of the hand path from a straight line to the target, (2) the perpendicular displacement of the hand path at the maximum tangential velocity, and (3) the area between the curve defined by the hand path and a straight line to the target.

\section{RESULTS}

The hypothesis was that the transfer of learning across arm configurations depends on the similarity in joint displacements and not on the similarity in hand displacements. In two experiments, we compared the performance of two groups of subjects: group $\mathrm{j}$, for which the training target involved the same joint displacement as the transfer target, and group $\mathrm{h}$, for which the training target corresponded to the same hand displacement.

We analyzed data from the first and last sets of movements in the training phase and from the transfer test. For each subject, we computed scores for each kinematic index by averaging the measures obtained on the initial three trials of each of these sets. The 
A

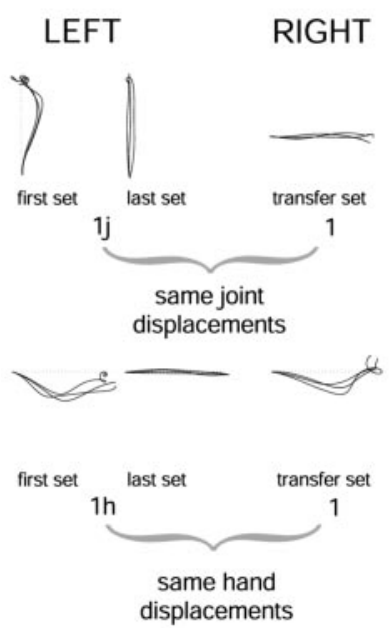

B

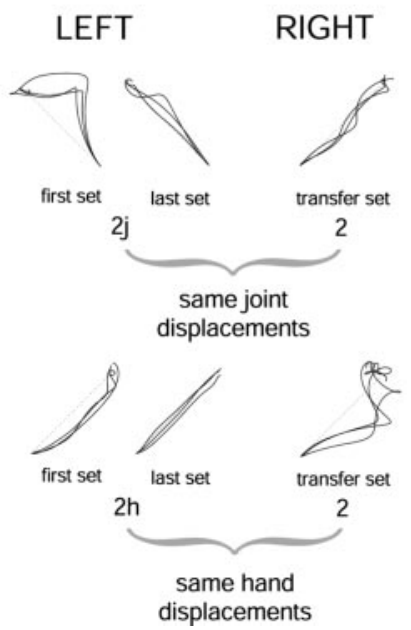

Figure 2. Transfer of learning between workspace locations is observed for movements involving the same joint displacements. No transfer is observed when movements in different locations are matched in terms of hand displacement. $A$ and $B$ give results for single subjects in experiments 1 and 2 . In each case, training trials at the left of the workspace and transfer trials at the right are shown.

analyses were repeated using values from all 20 movements in the first, last, and transfer sets and led to similar results.

\section{Experiment 1}

The subjects in group $\mathrm{h}$ trained to make movements to target $1 \mathrm{~h}$; the subjects in group $\mathrm{j}$ reached to target $1 \mathrm{j}$. Figure $2 A$ shows data for two different subjects in group $\mathrm{j}$ (top) and group h (bottom), respectively. The left shows the first three trials of the first and last training sets at the left of the workspace. The right shows the hand paths for the first three movements of the transfer set at the right.

Figure 2 shows that initial exposure to the force field had comparable effects in both conditions; the first movements substantially deviated from a straight line from the center to the training target. By the end of training, the hand paths were quite straight for both groups of subjects, indicating comparable levels of compensation.

Although the movements for all subjects were substantially affected when they first encountered the force field at the left, the subjects in the two groups exhibited different levels of adaptation when they first interacted with the field in the new configuration at the right. Specifically, in the transfer trials, the hand paths were straight when training and transfer involved the same joint displacements. Paths in the transfer trials were deviated when the training and transfer trials had the same hand displacements. In fact, the hand paths of this latter group of subjects were as deviated as in the initial exposure to the force field at the left in the training configuration.

Figure $3 A$ shows mean values across subjects for each kinematic error measure. The white and gray bars denote groups $\mathrm{j}$ and $\mathrm{h}$, respectively. Performance is shown for the first and last blocks of training and for the transfer trials. Statistical tests were conducted using repeated-measures ANOVA. Post hoc Tukey tests examined specific pairwise differences. A reliable learning effect (between the first and last training blocks) was observed for groups $\mathrm{j}$ and $\mathrm{h}(p<0.01)$. Moreover, kinematic error was similar for groups $\mathrm{j}$ and $\mathrm{h}$ at the beginning of training $(p>0.05)$ and also at the end of training $(p>0.05)$. Kinematic error in the transfer test

A

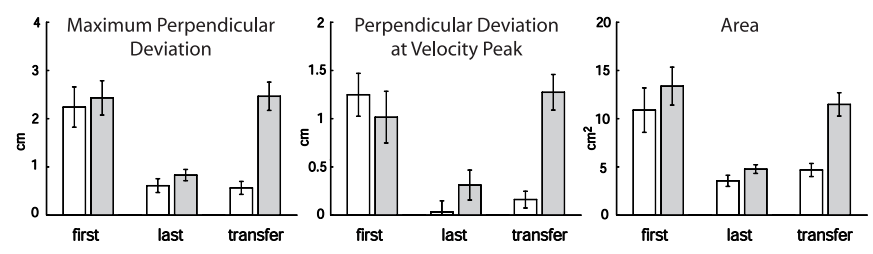

B

Maximum Perpendicular Deviation
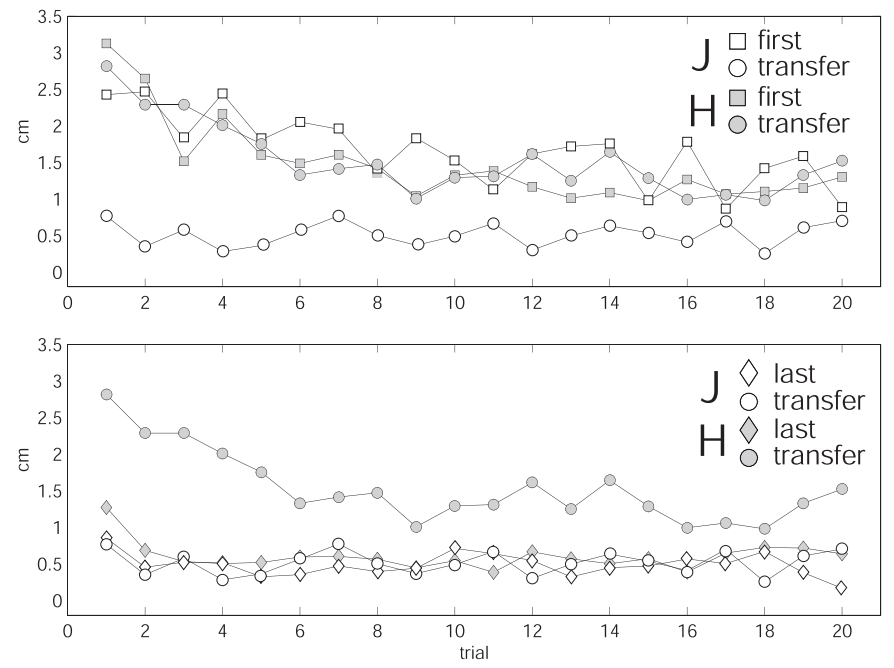

Figure 3. A, Mean values across subjects (experiment 1) of kinematic error for the first and last training trials and for the transfer trials. Error bars denote \pm 1 SE. Gray bars are for group h; white bars are for group j. $B$, Mean values across subjects for each of the 20 trials in the first and last training sets and the transfer set. In both panels transfer is observed when movements involve the same joint displacements.

was greater for group $\mathrm{h}(p<0.01)$. For subjects in group $\mathrm{j}$ for whom joint displacements were similar in the two workspace locations, kinematic error in the transfer trials was comparable with the error observed at the end of training $(p>0.05)$. In contrast, when hand displacements were similar in transfer and training (group h), the error in transfer trials was as large as that observed on initial exposure to the force field in the other arm configuration $(p>0.05)$. Comparable statistical results were obtained for all three measures of kinematic error.

\section{Experiment 2}

The subjects in group $\mathrm{h}$ were trained to make movements to target $2 \mathrm{~h}$; the subjects in group $\mathrm{j}$ moved to target $2 \mathrm{j}$. Training and transfer targets for group $\mathrm{h}$ involved identical hand displacements. For group j, training and transfer targets involved the same joint displacements. As can be seen in Figure 1, the training target for group $\mathrm{j}$ involved hand displacements that were orthogonal to those required for the transfer target.

In contrast to experiment 1 , in which the joint paths involved in the two training conditions were not orthogonal but were of comparable lengths, in experiment 2 the joints paths were orthogonal but differed in length (the joint path to target $2 \mathrm{j}$ was longer than to target $2 \mathrm{~h}$ ) (Fig. 1). Because movement durations were constant, this resulted in smaller training torques for the subjects in group $\mathrm{h}$. The effect is illustrated in Figure $2 B$, which shows individual data. When first encountering the field at the left, the subject in group $\mathrm{j}$ (Fig. 1, top) had his hand more deviated than 
the subject in group h (Fig. 1, bottom). At the end of training, the lateral deviation of the hand path had diminished in both conditions. The performance of each subject in the transfer test was similar to that observed in experiment 1 . That is, the subjects in group $\mathrm{j}$ showed better transfer of adaptation across arm configurations than the subjects in group h. For group $\mathrm{j}$, kinematic error at the end of the training and in the transfer test were of comparable magnitude, whereas for group $h$ the hand path deviated substantially in the transfer condition. A significant learning effect between the first and last training blocks was observed for group $\mathrm{j}$ alone $(p<0.01)$. As explained in Materials and Methods, training torques were less for group $h$. This presumably contributed to the reduction in the training effect. At the end of training, there were no reliable differences between the two groups in kinematic error $(p>0.05)$. As in experiment 1 , the error in the transfer task was significantly greater for group $h$ than for group $\mathrm{j}(p<0.01)$. For group $\mathrm{j}$, there were no differences in error between the end of the training and transfer task trials $(p>0.05)$, whereas for group $\mathrm{h}$ the error increased in the transfer trials $(p<$ 0.01). Similar results were obtained for all three measures.

Figure $3 B$ shows the means across subjects of the maximum perpendicular deviation for each of the 20 trials of the first and last training sets and the transfer set. White and gray symbols represent groups $\mathrm{j}$ and $\mathrm{h}$, respectively. In Figure $3 B$, top, the transfer trials (circles) are compared with the first training trials (squares). In Figure 3B, bottom, the transfer trials (circles) are compared with the last training trials (diamonds). The data are for experiment 1 , but the same trends are seen in experiment 2.

In Figure $3 B$, top, it can be seen that the hand-path deviation for group $\mathrm{j}$ decreased markedly over the initial trials at the beginning of training (white squares). In contrast, in the transfer set, the curve for group $\mathrm{j}$ (white circles) is flat. The hand-path deviation is initially small and remains so throughout the 20 trials. In contrast, for the subjects in group h (gray symbols), the curves that correspond to movements made in different arm configurations are similar in shape: deviation decreases steadily from the first to the 20th movement (and is similar to the curve for group $\mathrm{j}$ at the beginning of training). In other words, the subjects in group $h$ exhibit a pattern of error that indicates that no transfer of learning occurred. In the new limb configuration, the subjects of group $\mathrm{h}$ (gray circles) had to start the adaptation process from scratch. This was so despite the fact that the hand trajectory and force field were the same in the two limb configurations.

Figure $3 B$, bottom, shows that for group $\mathrm{j}$ (white symbols) the curves for the final training trials and transfer trials overlap. For group h (gray symbols), the performance in transfer trials is worse than in the final training trials. For subjects in group $\mathrm{j}$, who trained making movements in a direction that was orthogonal in hand space to the transfer trajectory, changing arm configuration and hand trajectory had little effect on adaptation.

\section{Directional asymmetries in force-field effects}

An examination of Figure 1 shows that movements in different directions that are equal in amplitude in hand space translate into joint paths of different lengths. Consequently, equal forces at the hand translate into torques at the joints of unequal magnitude. As a result, one would expect different perturbing effects of the field depending on the movement direction and workspace location. As noted above, two separate studies were needed to enable, first, the examination of movements that were equated in terms of the experienced forces and torques and, second, the examination of movements that were orthogonal in both hand and joint space.

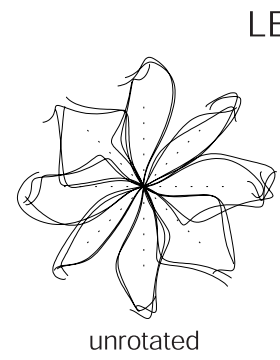

LEFT
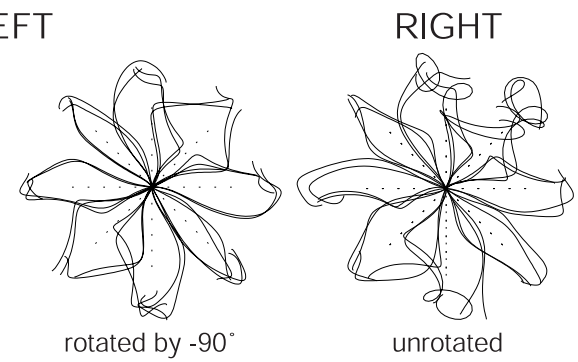

Figure 4. Asymmetries in the effect of the force field are observed for movements in different directions. Shown are movement paths for centerout movements in which a clockwise or counterclockwise curl field is unexpectedly introduced. The effects at the left and right of the workspace are similar once they are corrected for the angle at the shoulder.

This situation is characteristic of a dependence of hand-path deviation in force fields on movement direction in conjunction with workspace location.

As a control procedure, to assess the effect of the field in different directions, we examined the pattern of deviations attributable to the initial exposure to the force field when subjects made movements in different directions. Figure 4 shows data for centerout movements to eight targets in the left and right workspace locations. The amplitude and duration of the movements were the same as in the experiments described above. In $12.5 \%$ of pseudorandomly selected movements, forces were applied by the robot. The remainder were performed in null-field conditions. The force field was clockwise for half of these field-on trials and counterclockwise for the other half.

Figure 4 shows how the hand deviated during the force-field trials in the two configurations (unrotated). The center corrects for differences between left and right workspace locations in the angle at the shoulder by rotating the pattern at the left clockwise by $90^{\circ}$. In both left and right workspace locations there are asymmetries in the effect of the force field on different movement directions. An examination of Figure 1 shows that the asymmetries are directly related to the length of the path in joint space and accordingly to the magnitude of the associated torques. A comparison of the rotated pattern with the pattern observed in the right arm configuration indicates that the effect of the field was similar in the two locations once corrected for the angle at the shoulder. That is, a similar pattern of deviation is observed on initial exposure to the force field at both workspace locations as long as the joint excursions are the same (which is the case after a $90^{\circ}$ clockwise rotation about the shoulder).

\section{DISCUSSION}

We have examined how the learning of dynamics generalizes across arm configurations with the goal of distinguishing the coding of movements within an extrinsic frame of reference from the coding within an intrinsic system of coordinates. By completely dissociating the influence on the transfer of the similarity in joint displacement from the similarity in hand direction, we provide a simple and direct demonstration that the transfer of learning depends on the similarity at the level of the joints. These results are consistent with the findings of Shadmehr and MussaIvaldi (1994) and Ghez et al. (2000); they provide support for the idea that limb dynamics are represented with a joint- or musclebased coordinate system.

The present study resolves a concern regarding previous evidence favoring intrinsic movement coding. Shadmehr and Mussa- 
Ivaldi (1994) demonstrated that invariance in a torque field determines the transfer rather than an invariance in the forces applied at the hand. However, in that experiment, subjects had to learn a hand velocity-dependent force field in which the directions of the forces applied at the hand rotate in a complex way, from assisting to resisting the movement, with changes in the direction of the target. The field used in the present study acts in all cases perpendicular to the direction of movement. Even under these conditions, in which there is a simple mapping between the direction of hand movement and the direction of the force at the hand, the transfer of learning depends on the similarity of joint displacement.

By using movements in a single direction, rather than multiple directions, as is often the case in studies of force-field learning, the exact mapping of learning between the training and testing locations could be explicitly specified. The present results are consistent with the idea that learning is local (Gandolfo et al., 1996; Thoroughman and Shadmehr, 2000). Transfer was found to be maximum for the same direction of joint displacement and absent for dissimilar directions of joint motion. This suggests that generalizable knowledge of dynamics involves a composition of many specific adaptations of control that are needed to achieve movements in different directions.

Previous claims regarding the local nature of dynamics learning have been based on the examination of generalization across directions within a single workspace location; the angular distance between movements was expressed in hand space. The directional asymmetries shown in Figure 4 raise concerns regarding the examination of generalization in hand space. It is seen that hand-path deviation differs for movements in different directions as a result of differences in joint excursion and, hence, the amplitude of the resulting torques. This complicates the interpretation of generalization across hand-movement directions. The decay of adaptation with angular distance from the training direction is confounded with differential field-strength effects for movements in surrounding directions. Patterns of generalization in hand space may as a result be a poor model on which to draw conclusions regarding this process.

In the present study, we examined the generalization of dynamics learning by using a curl field. The present finding of the transfer of adaptation to new dynamics based on the similarity of joint displacements also seems to hold for the fields used by Shadmehr and Mussa-Ivaldi (1994) and for inertial loads (Ghez et al., 2000). Nevertheless, the generality of the finding remains to be tested in conditions that could prompt extrinsic encoding, such as adaptation to forces applied in directions that are independent of the motion of the limb.

\section{REFERENCES}

Gandolfo F, Mussa-Ivaldi FA, Bizzi E (1996) Motor learning by field approximation. Proc Natl Acad Sci USA 93:3843-3846.

Ghez C, Krakauer JW, Sainburg RL, Ghilardi M-F (2000) Spatial representations and internal models of limb dynamics in motor learning. In: The new cognitive neurosciences (Gazzaniga MS, ed), pp 501-514. Cambridge, MA: MIT.

Hemminger SE, Donchin O, Ariff GD, Young ED, Shadmehr R (2001) Intermanual generalization of arm dynamics in extrinsic coordinates. Soc Neurosci Abstr 27:302.1.

Lackner JR, Dizio P (1994) Rapid adaptation to coriolis force perturbations of arm trajectory. J Neurophysiol 72:299-313.

Morton SM, Lang CE, Bastian AJ (2001) Inter- and intra-limb generalization of adaptation during catching. Exp Brain Res 141:438-445.

Sainburg RL, Ghez C, Kalakanis D (1999) Intersegmental dynamics are controlled by sequential anticipatory, error correction, and postural mechanisms. J Neurophysiol 81:1045-1056. arsid6318315

Shadmehr R, Moussavi ZM (2000) Spatial generalization from learning dynamic of reaching movements. J Neurosci 20:7807-7815. arsid6318315

Shadmehr R, Mussa-Ivaldi FA (1994) Adaptive representation of dynamics during learning of motor task. J Neurosci 14:3208-3224.

Thoroughman KA, Shadmehr R (2000) Learning through adaptive combination of motor primitives. Nature 407:742-747. 